\title{
HERMITE FUNCTION EXPANSIONS VERSUS HERMITE POLYNOMIAL EXPANSIONS
}

\author{
I. ABU-FALAHAH and J. L. TORREA* \\ Departamento de Matemáticas Facultad de Ciencias Universidad Autónoma \\ de Madrid 28049 Madrid, Spain \\ e-mail: ibraheem.abufalahah@uam.es, joseluis.torrea@uam.es
}

(Received 5 May, 2005; accepted 15 March, 2006)

\begin{abstract}
We consider expansions with respect to the multi-dimensional Hermite functions and to the multi-dimensional Hermite polynomials. They are respectively eigenfunctions of the Harmonic oscillator $\mathcal{L}=-\Delta+|x|^{2}$ and of the OrnsteinUhlenbeck operator $\mathbf{L}=-\Delta+2 x \cdot \nabla$. The corresponding heat semigroups and Riesz transforms are considered and results on both aspects (polynomials and functions) are obtained.
\end{abstract}

2000 Mathematics Subject Classification. 42C10, 42B20, 42B25.

1. Introduction. We shall work in the space $\mathbb{R}^{d}$, endowed either with the Lebesgue measure $d x$ or with the Gaussian measure $d \gamma(x)=\pi^{-d / 2} e^{-|x|^{2}} d x$. Consider the system of multidimensional Hermite polynomials

$H_{\alpha}(x)=H_{\alpha_{1}}\left(x_{1}\right) \cdot \ldots \cdot H_{\alpha_{d}}\left(x_{d}\right), \quad x=\left(x_{1}, \ldots, x_{d}\right), \quad \alpha=\left(\alpha_{1}, \ldots \alpha_{d}\right), \alpha_{i} \in\{0,1, \ldots\}$,

where $H_{k}(s)=(-1)^{k} e^{s^{2}} \frac{d^{k} e^{-s^{2}}}{d s^{k}}, s \in \mathbb{R}$, denotes the 1-dimensional $k$ th Hermite polynomial, see [18]. It is well known that the Hermite polynomials are the eigenfunctions of the Ornstein-Uhlenbeck differential operator $\mathbf{L}=-\Delta+2 x \cdot \nabla$, namely

$$
\mathbf{L} H_{\alpha}=2|\alpha| H_{\alpha}, \quad|\alpha|=\alpha_{1}+\cdots+\alpha_{d} .
$$

The operator $\mathbf{L}$ is positive and symmetric in $L^{2}\left(\mathbb{R}^{d}, d \gamma(x)\right)$ on the domain $C_{c}^{\infty}\left(\mathbb{R}^{d}\right)$. The orthonormalized Hermite polynomials, $\tilde{H}_{k}=\frac{2^{-k / 2}}{\sqrt{k !}} H_{k}$ form an orthonormal basis for $L^{2}(d \gamma(x))$.

We shall also consider the system of multidimensional Hermite functions

$$
h_{\alpha}(x)=h_{\alpha_{1}}\left(x_{1}\right) \cdot \ldots \cdot h_{\alpha_{d}}\left(x_{d}\right), \quad x=\left(x_{1}, \ldots, x_{d}\right), \quad \alpha=\left(\alpha_{1}, \ldots \alpha_{d}\right), \alpha_{i} \in\{0,1, \ldots\},
$$

where $h_{k}(s)=\left(\pi^{1 / 2} 2^{k} k !\right)^{-1 / 2} H_{k}(s) e^{-s^{2} / 2}$ and $H_{k}$ denotes the 1-dimensional $k$ th Hermite polynomial. It is well known that the Hermite functions are the eigenfunctions of the

\footnotetext{
*Second author was partially supported by the HARP network HPRN-CT-2001-00273 of the European Commission and by grant MTM2005-08350-C03-01 of Ministerio de Educación y Ciencia.
} 
Hermite differential operator $\mathcal{L}=-\Delta+|x|^{2}$, namely

$$
\mathcal{L} h_{\alpha}=(2|\alpha|+d) h_{\alpha}
$$

The functions $h_{\alpha}$ form an orthonormal basis for $L^{2}\left(\mathbb{R}^{n}, d x\right)$. The operator $\mathcal{L}$ is positive and symmetric in $L^{2}\left(\mathbb{R}^{d}, d x\right)$ on the domain $C_{c}^{\infty}\left(\mathbb{R}^{d}\right)$, see [19].

The Ornstein-Uhlenbeck, $e^{-t \mathbf{L}}$, (respectively the Hermite, $e^{-t \mathcal{L}}$,) semigroup with infinitesimal generator $-\mathbf{L}$ (respectively $-\mathcal{L}$ ) can be defined in a spectral sense. Namely for functions $g \in L^{2}(d \gamma(x))$ such that $g=\sum c_{\alpha} \tilde{H}_{\alpha}$ define $e^{-t \mathbf{L}} g$ the $L^{2}\left(e^{-|x|^{2}} d x\right)$ function given by $e^{-t \mathbf{L}} g=\sum e^{-2 t|\alpha|} c_{\alpha} \tilde{H}_{\alpha}$. On the other hand if $f \in L^{2}(d x)$ such that $f=\sum c_{\alpha} h_{\alpha}$ define $e^{-t \mathcal{L}} f$ be the $L^{2}(d x)$ function given by $e^{-t \mathcal{L}} f=\sum e^{-t(2|\alpha|+d)} c_{\alpha} h_{\alpha}$.

B. Muckenhoupt initiated in 1969 the study, in dimension one, of the maximal operator of the Ornstein-Uhlenbeck semigroup, $\sup _{t} e^{-t \mathbf{L}}$, and also the notion of "conjugate function" related to that semigroup, see [9], [10]. $e^{-t \mathbf{L}}$ is a symmetric diffusion semigroup, in the sense of [13], the $\left(L^{p}, L^{p}\right), 1<p<\infty$, boundedness of the maximal operator is deduced from the general theory developed in [13]. In finite dimensions, the proof of the $(1,1)$-weak boundedness for the maximal operator was given in 1982 by P. Sjögren, see [12]. The corresponding result for the Riesz transforms was proved by Fabes, Gutiérrez and Scotto in [4]. They also proved that the Riesz transforms are principal value operators. Due to the relation with Wiener chaos, proving that the constants appearing in the boundedness are independent of the dimension became an important task. Some research was done in this direction, see [5] and the references in the survey [17]. Finally, weighted inequalities and vector-valued inequalities were studied in [7] and [6].

As for the semigroup $e^{-t \mathcal{L}}$, the main reference is by Thangavelu. He proved (in several papers but we refer to his book, [19], and the references there) the $\left(L^{p}(d x), L^{p}(d x)\right)$ and $\left(L^{1}(d x), L^{1, \infty}(d x)\right)$ boundedness for the maximal operator of the semigroup. He also proved, by using analogues of the classical conjugate harmonic functions, that the Riesz transforms, see the definition in section 2 formula (6) and the comments there, are $\left(L^{p}(d x), L^{p}(d x)\right)$ and $\left(L^{1}(d x), L^{1, \infty}(d x)\right)$ bounded. This study was extended in [15] and weighted inequalities for the weights in the $A_{p}$-class of Muckenhoupt were proved. For an introduction to the $A_{p}$ theory see [3]. Neither in [19] nor in [15] was the description of the Riesz transforms as principal value operator considered.

There is a close relation between the semigroups $e^{-t \mathcal{L}}$ and $e^{-t \mathbf{L}}$. This relation, that is determined by the fact $h_{k}(s)=\left(\pi^{1 / 2} 2^{k} k !\right)^{-1 / 2} H_{k}(s) e^{-s^{2} / 2}$, is propagated to the operators defined through the semigroups (maximal operators, Riesz transforms, etc). This kind of correspondence between these operators is sometimes described vaguely (in this case) saying that the operators associated to $\mathcal{L}$ and $\mathbf{L}$ are "unitary equivalent in $L^{2}$ ". The purpose of this note is to describe, in a transparent and clear way, this relation and to get, as a consequence, new results for several operators associated either to $\mathbf{L}$ or to $\mathcal{L}$. The relationship between both parts is described in Proposition 3.3 and Theorem 3.5. By using these results we can get new weighted inequalities in both sides, see Theorems 2.1 and 2.5, we also get new descriptions of the Riesz transforms in the Hermite function case, see Theorem 2.2 and 2.3 .

The organization of the paper is the following. We present the results in Section 2. These results shall be proved in Section 4, with the help of some technical results that we present in Section 3. 
2. Main results. If $f$ is a linear combination of Hermite functions then $e^{-t \mathcal{L}} f(x)=$ $\int_{\mathbb{R}^{n}} G_{t}(x, y) f(y) d y$, where $G_{t}(x, y)$ is given by

$$
\begin{aligned}
G_{t}(x, y) & =\sum_{\alpha} e^{-t(2|\alpha|+d)} h_{\alpha}(x) h_{\alpha}(y) \\
& =(2 \pi \sinh 2 t)^{-d / 2} \exp \left(-\frac{1}{2}|x-y|^{2} \operatorname{coth} 2 t-x \cdot y \tanh t\right),
\end{aligned}
$$

see [19], [15]. Clearly $e^{-t(\mathcal{L}-d)} f(x)=\int_{\mathbb{R}^{n}} e^{t d} G_{t}(x, y) f(y) d y$

We have the following theorem.

THEOREM 2.1. Let $v$ be a positive measurable function. The following conditions are equivalent:

(i) There exists a positive measurable function $u$ and a constant $C$ such that for every $f \in L^{2}(v(x) d x)$ we have

$$
\sup _{t} \int_{\mathbb{R}^{d}}\left|e^{-t(\mathcal{L}-d)} f(x)\right|^{2} u(x) d x \leq C \int_{\mathbb{R}^{d}}|f(x)|^{2} v(x) d x .
$$

(ii) There exists a positive measurable function $u$ and a constant $C$ such that for every $f \in L^{2}(v(x) d x)$ we have

$$
\int_{\mathbb{R}^{d}} \sup _{t}\left|e^{-t(\mathcal{L}-d)} f(x)\right|^{2} u(x) d x \leq C \int_{\mathbb{R}^{d}}|f(x)|^{2} v(x) d x .
$$

(iii) The function $v$ satisfies $\int_{\mathbb{R}^{d}} v^{-1}(x) e^{-|x|^{2}} d x<\infty$.

In particular for a function $v$ satisfying (iii) the operator $\sup _{t} e^{-t \mathcal{L}}$ maps $L^{2}\left(\mathbb{R}^{d}, v(x) d x\right)$ into $L^{2}\left(\mathbb{R}^{d}, u(x) d x\right)$ for some positive $u$.

It is well known that $\mathcal{T}_{t}=e^{-t \mathcal{L}}$ (respectively $\mathbf{T}_{t}=e^{-t \mathbf{L}}$ ) is a diffusion semigroup in $L^{p}(d x), 1 \leq p \leq \infty$ (respectively in $\left.L^{p}(d \gamma(x)), 1 \leq p \leq \infty\right)$ in the sense of [13], see [17], [19] and [15] for details.

The operators $e^{-t \mathbf{L}}$ and $e^{-t \mathcal{L}}$ are positive, $\left(f(x) \geq 0 \rightarrow e^{-t \mathbf{L}} f(x) \geq 0\right)$, bounded in $L^{p}(d \gamma(x))$ (respectively $L^{p}(d x)$ ) and therefore each $\mathbf{T}_{t}$ (respectively each $\mathcal{T}_{t}$ ) have a straightforward extension to $L_{B}^{p}(d \gamma(x))$ (respectively $L_{B}^{p}(d x)$ ) for every Banach space $B$. Moreover the norm of the extension is the same as the original norm of the operator. By $L_{B}^{p}(d \gamma(x))$ we denote the Bochner-Lebesgue space of $B$-valued functions defined in $\mathbb{R}^{n}$ such that $\int_{\mathbb{R}^{n}}\|f(x)\|_{B}^{p} d \gamma(x)<\infty$. Analogous definitions can be given for $L_{B}^{p}(d x)$. Since these extensions are linear, they act in a natural way over the tensor products $B \otimes L^{p}(d \gamma(x))$ and $B \otimes L^{p}(d x)$. In particular

$$
\mathbf{T}_{t}\left(\sum_{i=1}^{n} b_{i} \varphi_{i}\right)=\sum_{i=1}^{n} b_{i} \mathbf{T}_{t} \varphi_{i}, \quad b_{i} \in B, \varphi_{i} \in L^{p}(d \gamma(x))
$$

Analogous expressions can be given for $\mathcal{T}_{t}$.

Let $\mu$ be a $\sigma$-finite measure in $\mathbb{R}^{n}$. Let $\left\{T_{t}\right\}$ be a symmetric diffusion semigroup of operators acting on measurable functions on $\left(\mathbb{R}^{n}, d \mu\right)$, with a second order differential operator $-L$, (symmetric in $L^{2}(d \mu)$ ) as its infinitesimal generator. In this context, the following operators can be considered; see [13], 
Riesz potentials:

$$
\text { given } \quad a>0, \quad(-L)^{-a} f(x)=\frac{1}{\Gamma(a)} \int_{0}^{\infty} t^{a-1} T_{t} f(x) d t .
$$

\section{Riesz transforms:}

$$
\text { For } \quad 1 \leq i \leq n, \quad R_{i} f(x)=\text { " } \frac{\partial}{\partial x_{i}} " L^{-1 / 2} f(x) .
$$

Here " $\frac{\partial}{\partial x_{i}}$ " denotes the component of the "gradient" associated to the operator $L$. It is easy to check that

$$
\mathbf{L}=\sum_{j=1}^{n} \delta_{j}^{*} \delta_{j}, \quad \text { where } \quad \delta_{j}=\frac{\partial}{\partial x_{j}} \text { and } \delta_{j}^{*}=-\frac{\partial}{\partial x_{j}}+2 x_{j} .
$$

Observe that $\delta_{j}^{*}$ is the adjoint operator of $\delta_{j}$ in $L^{2}(d \gamma(x))$-sense. In a parallel way we have

$$
\mathcal{L}=\frac{1}{2} \sum_{j=1}^{n}\left(A_{j}^{*} A^{*}+A_{j} A_{j}^{*}\right), \text { where } A_{j}=\frac{\partial}{\partial x_{j}}+x_{j}, \text { and } A_{j}^{*}=-\frac{\partial}{\partial x_{j}}+x_{j}
$$

Observe that $A_{j}^{*}$ and $A_{j}$ are adjoints in $L^{2}(d x)$-sense.

Therefore the operator " $\frac{\partial}{\partial x_{i}}$ " will be, $\delta_{i}$ in the case of $\mathbf{L}$, and either $A_{i}$ or $A_{i}^{*}$ in the case of the operator $\mathcal{L}$.

Since 0 is an eigenvalue of $\mathbf{L}$, the negative powers $\mathbf{L}^{-a}$ are not defined for every function in $L^{2}\left(\mathbb{R}^{n}, d \gamma(x)\right)$. Let $\Pi_{0}$ be the orthogonal projection onto the orthogonal complement of the eigenspace correponding to the eigenvalue 0 . Then the Riesz transforms for the Ornstein-Uhlenbeck operator are defined as, see [17], $\mathbf{R}_{i}=\delta_{i}(\mathbf{L})^{-1 / 2} \Pi_{0}$, in particular in defining $\mathbf{R}_{i} f$ we always can assume that $\int_{\mathbb{R}^{n}} f(x) d \gamma(x)=0$. As we said it is known that $\mathbf{R}_{i}$ are bounded from $L^{p}\left(\mathbb{R}^{n}, d \gamma\right)$ into itself for $p$ in the range $1<p<\infty$, and from $L^{1}\left(\mathbb{R}^{n}, d \gamma\right)$ into $L^{1, \infty}\left(\mathbb{R}^{n}, d \gamma\right)$. They are principal value operators, that is

$$
\mathbf{R}_{i} f(x)=\lim _{\varepsilon \rightarrow 0} \int_{|x-y|>\varepsilon} \mathbf{R}_{i}(x, y) f(y) d \gamma(y) \text {, a.e. } x, \quad f \in L^{1}(d \gamma) .
$$

See the survey [17] and the references there.

In the case of $\mathcal{L}$, the Riesz transforms, due to (8), were defined, see [19], as $\mathcal{R}_{i}^{+}=$ $A_{i}(\mathcal{L})^{-1 / 2}$ and $\mathcal{R}_{i}^{-}=A_{i}^{*}(\mathcal{L})^{-1 / 2}$. For these we shall prove the following theorem.

THEOREM 2.2. The operators $\mathcal{R}_{i}^{ \pm}, i=1, \ldots, n$ are principal valued operators. That is

$$
\mathcal{R}_{i}^{ \pm} f(x)=\lim _{\varepsilon \rightarrow 0} \int_{|x-y|>\varepsilon} \mathcal{R}_{j}^{ \pm}(x, y) f(y) d y, \quad f=\sum_{\text {finite }} c_{\alpha} h_{\alpha} .
$$

The operators $\delta_{i}^{*}(\mathbf{L})^{-1 / 2} \Pi_{0}, i=1, \ldots, n$ are principal value operators over the class of polynomial functions.

We call $\mathcal{R}_{i}^{ \pm}$the linear extension of these operators, in the sense described in (4), to functions taking values in a Banach space $B$. We recall that a Banach space is in the 
$U M D$ class if the Hilbert transform has a bounded extension to $L_{B}^{2}(\mathbb{R}, d x)$, see [2] and [1]. We have the following theorem.

THEOREM 2.3. Let $B$ be a Banach space. The following statements are equivalent:

(i) $B$ is a UMD Banach space.

(ii) $\left|\left\{x \in \mathbb{R}^{n}:\left\|\mathcal{R}_{j}^{+} f(x)\right\|_{B}>\lambda\right\}\right| \leq \frac{C}{\lambda} \int_{\mathbb{R}^{n}}\|f(x)\|_{B} d x, 1 \leq j \leq n$.

(iii) For every $p, 1<p<\infty$ (and equivalently for some $1<p<\infty$ ),

$$
\left\|\mathcal{R}_{j}^{+} f\right\|_{L_{B}^{p}(d x)} \leq C_{p}\|f\|_{L_{B}^{p}(d x)}, \quad 1 \leq j \leq n
$$

(iv) $\mathcal{R}_{j}^{+}$maps boundedly $L_{B}^{\infty}$ into $B M O_{B}, 1 \leq j \leq n$.

(v) $\mathcal{R}_{j}^{+}$maps $H_{B}^{1}$ into $L_{B}^{1}, 1 \leq j \leq n$.

In (ii), (iii), (iv) and (v), $\mathcal{R}_{j}^{+}$can be replaced by $\mathcal{R}_{j}^{-}$. The constants $C$ and $C_{p}$ are independent off but they may depend on the Banach space $B$.

Moreover if $B$ is a UMD Banach space then, for $1 \leq j \leq n$,

$$
\mathcal{R}_{j}^{ \pm} f(x)=\lim _{\varepsilon \rightarrow 0} \mathcal{R}_{j, \varepsilon}^{ \pm} f(x)=\lim _{\varepsilon \rightarrow 0} \int_{|x-y|>\varepsilon} \mathcal{R}_{j}^{ \pm}(x, y) f(y) d y \text {, a.e. } x, f \in \cup_{1 \leq p \leq \infty} L_{B}^{p}(d x) .
$$

REMARK 2.4. Observe that in the above theorem we said that $\mathcal{R}_{j}^{ \pm}$are defined in $L^{\infty}\left(\mathbb{R}^{n}, d x\right)$. This is different from the case of the classical euclidean Riesz transforms for which a definition for $L^{\infty}$ functions has to be given "ad hoc", see [14]. To justify this fact it is enough to see that for a function $f \in L^{\infty}$, the limit

$$
\lim _{n \rightarrow \infty}\left(\mathcal{R}_{j}^{+}\left(f \chi_{B(0, n)}\right)(x)+\int_{|y|>n} \mathcal{R}_{j}^{+}(x, y) f(y) d y\right)
$$

exists a.e. $x$. In order to prove the existence of this limit we need two ingredients: first the existence of the limit for functions in $L^{p}$ (this is the case of $f \chi_{B(0, n)}$ ), second, the bound $\mathcal{R}_{j}^{+}(x, y) \leq C e^{-\frac{|x-y|^{2}}{c}}$ for $|x-y|>1$, see Proposition 3.4, guarantees the convergence of the second summand.

The Riesz transforms $\mathcal{R}_{j}^{ \pm}$are Calderón-Zygmund operators with associated Calderón-Zygmund kernels $\mathcal{R}_{j}^{ \pm}(x, y)$, see [15], in the sense that

$$
\mathcal{R}_{i}^{ \pm} f(x)=\int_{\mathbb{R}^{n}} \mathcal{R}_{j}^{ \pm}(x, y) f(y) d y, \quad f \in C_{c}^{\infty}\left(\mathbb{R}^{n}\right), \quad x \notin \sup f .
$$

Therefore they are bounded from $L^{p}\left(\mathbb{R}^{n}, \omega(x) d x\right)$ into itself for $p$ in the range $1<$ $p<\infty$ and from $L^{1}\left(\mathbb{R}^{n}, \omega(x) d x\right)$ into $L^{1, \infty}\left(\mathbb{R}^{n}, \omega(x) d x\right)$, where $\omega$ is a weight in the Muckenhoupt $A_{p}$-class, $1 \leq p<\infty$. We have the following theorem.

THEOREM 2.5. Let $\omega$ be a weight in Muckenhoupt class $A_{2}$. The operators $\mathbf{R}_{i}$ and $\delta_{i}^{*}(\mathbf{L})^{-1 / 2} \Pi_{0}, i=1, \ldots, n$ are bounded from $L^{2}\left(\mathbb{R}^{n}, \omega(x) d \gamma(x)\right)$ into itself.

3. Technical Lemmas. We define in the following simple lemma the key operator which shall be the carrier of the results from the polynomial side to the function side and vice-versa.

LEMMA 3.1. Let B be a Banach space and $\omega$ a weight in $\mathbb{R}^{n}$. The operator $U$ defined by $U f(x)=f(x) \pi^{d / 4} e^{-\frac{|x|^{2}}{2}}$, is an isometry from $L_{B}^{2}(\omega(x) d \gamma(x))$ into $L_{B}^{2}(\omega(x) d x)$. 
Proof.

$$
\begin{aligned}
\|U f\|_{L_{B}^{2}(\omega(x) d x)}^{2} & =\int\|U f(x)\|_{B}^{2} \omega(x) d x=\int\|f(x)\|_{B}^{2} \pi^{d / 2} e^{-|x|^{2}} \omega(x) d x \\
& =\|f\|_{\left.L_{B}^{2} \omega(x) d \gamma(x)\right)}^{2} .
\end{aligned}
$$

Definition 3.2. Let $B$ a Banach space . Let $H_{k}$ be the Hermite polynomials in $\mathbb{R}^{d}$. Any function $f$ of the form $f(x)=\sum_{\text {finite }} b_{\alpha} H_{\alpha}, x \in \mathbb{R}^{d}$, where $b_{\alpha} \in B$, will be called " $B$-valued polynomial function".

PROPOSITION 3.3. Let $B$ be a Banach space and f be a B-valued polynomial function in $\mathbb{R}^{n}$. We have the following pointwise identities

(i) $A_{j} U f(x)=U \delta_{j} f(x), \quad A_{j}^{*} U f(x)=U \delta_{j}^{*} f(x)$.

(ii) $(\mathcal{L}-d) U f(x)=U \mathbf{L} f(x), \quad \mathcal{L} U f(x)=U(\mathbf{L}+d) f(x)$.

(iii) $e^{-t(\mathcal{L}-d)} U f(x)=U e^{-t \mathbf{L}} f(x), \quad e^{-t(\mathcal{L})} U f(x)=U e^{-t(\mathbf{L}+d)} f(x)$.

(iv) Let $s>0$.

If $\int_{\mathbb{R}^{n}} f(x) d \gamma(x)=0$ then $(\mathcal{L}-d)^{-s} U f(x)=U(\mathbf{L})^{-s} f(x)$.

For every $f,(\mathcal{L})^{-s} U f(x)=U(\mathbf{L}+d)^{-s} f(x)$.

(v) If $\int_{\mathbb{R}^{n}} f(x) d \gamma(x)=0$ then $A_{i}(\mathcal{L}-d)^{-1 / 2} U f(x)=U \mathbf{R}_{i} f(x)$.

For every $f$ we have $\mathcal{R}_{i}^{+} U f(x)=U \delta_{i}(\mathbf{L}+d)^{-1 / 2} f(x), i=1, \ldots, n$.

We use the notations in (7) and (8).

Proof. (i) and (ii) are tedious calculations. By using (1), (2) we get (iii). By using the definition of Riesz potentials, it is very easy to check that $\mathbf{L}^{-s} H_{k}=(2|k|)^{-s} H_{k}$ and $\mathcal{L}_{H}^{-s} h_{k}=(2|k|+d)^{-s} h_{k}$. Observe that a polynomial function $f$ belongs to $\Pi_{0}$ when $\int_{\mathbb{R}^{d}} f(x) e^{-|x|^{2}} d x=0$. Finally, by using (7) and (8) we get (v).

The size of the kernels involved with the Riesz transforms where analyzed in Theorem 3.3 of [15]. In fact the following result is proved there.

Proposition 3.4. Let $f$ be a finite combination of Hermite functions. Then

(i) There exists a positive kernel L such that

$$
\left|\mathcal{L}^{-1 / 2} f(x)\right| \leq \int_{\mathbb{R}^{d}} L(x, y)|f(y)| d y, x \in \mathbb{R}^{d}
$$

If $d=1$ there exists an $\varepsilon, 0<\varepsilon<1$ with

If $d>1$, there exists a constant $C$ with

$$
L(x, y) \leq C\left(\frac{1}{|x-y|^{\varepsilon}} \chi_{|x-y|<1}+e^{-\frac{|x-y|^{2}}{c}} \chi_{|x-y|>1}\right) .
$$

$$
L(x, y) \leq C\left(\frac{1}{|x-y| d-1} \chi_{|x-y|<1}+e^{-\frac{|x-y|^{2}}{c}} \chi_{|x-y|>1}\right) .
$$

(ii) There exist constants $c, C$ such that

$$
\left|\mathcal{R}_{j}^{ \pm}(x, y)\right| \leq C\left(\frac{1}{|x-y|^{d}} \chi|x-y|<1+e^{-\frac{|x-y|^{2}}{c}} \chi|x-y|>1\right)
$$

The Proposition 3.3 suggest us to study the difference $(\mathcal{L})^{-1 / 2}-(\mathcal{L}-d)^{-1 / 2}$.

THEOREM 3.5. There exist kernels $N, L_{i}, i=1, \ldots, n$ such that for any function $f$ which is a linear combination of Hermite functions, with $\int_{\mathbb{R}^{n}} f(y) e^{-\frac{y^{2}}{2}} d y=0$ we have 
(i) $\left((\mathcal{L}-d)^{-1 / 2}-\mathcal{L}^{-1 / 2}\right) f(x)=\int_{\mathbb{R}^{d}} N(x, y) f(y) d y, x \in \mathbb{R}^{d}$.

(ii) $\left(A_{i}(\mathcal{L}-d)^{-1 / 2}-\mathcal{R}_{i}^{+}\right) f(x)=\int_{\mathbb{R}^{d}} L_{i}^{+}(x, y) f(y) d y, x \in \mathbb{R}^{d}, \quad i=1, \ldots n$.

(iii) $\left(A_{i}^{*}(\mathcal{L}-d)^{-1 / 2}-\mathcal{R}_{i}^{-}\right) f(x)=\int_{\mathbb{R}^{d}} L_{i}^{-}(x, y) f(y) d y, x \in \mathbb{R}^{d}, \quad i=1, \ldots n$.

Moreover there exist a one variable positive decreasing function $\Phi \in L^{1}(\mathbb{R}, d x)$ such that if we denote by $M$ either the kernel $N$ or the kernel $L_{i}^{ \pm}, \quad i=1, \ldots, n$, we have $|M(x, y)| \leq C \Phi(|x-y|)$.

Proof. Observe that the change of parameter

$$
t=t(s)=\frac{1}{2} \log \frac{1+s}{1-s}, \quad 0<s<1, \quad 0<t<\infty
$$

produces

$$
G_{t}(x, y)=K_{s}(x, y)=\left(\frac{1-s^{2}}{4 \pi s}\right)^{d / 2} \exp \left(-\frac{1}{4}\left(s|x+y|^{2}+\frac{1}{s}|x-y|^{2}\right)\right) .
$$

where $G_{t}$ is the kernel in (3). On the other hand, this change of parameter in (5) leads us to the expression

$$
L^{-1 / 2} f=\frac{1}{\Gamma(1 / 2)} \int_{0}^{1}\left(\log \frac{1+s}{1-s}\right)^{-1 / 2} e^{-t(s) L} f \frac{d s}{1-s^{2}} .
$$

Therefore for functions $f$ satisfying $\int_{\mathbb{R}^{n}} f(y) e^{-\frac{|y|^{2}}{2}} d y=0$ we have

$$
\begin{aligned}
\mathcal{L}^{-1 / 2} f= & \frac{1}{\Gamma(1 / 2)} \int_{0}^{1}\left(\log \frac{1+s}{1-s}\right)^{-1 / 2} \int_{\mathbb{R}^{n}} K_{s}(x, y) f(y) d y \frac{d s}{1-s^{2}} \\
= & \frac{1}{\Gamma(1 / 2)} \int_{0}^{1} \int_{\mathbb{R}^{n}}\left(K_{s}(x, y)-\chi_{(1 / 2,1)}(s)\left(\frac{1-s^{2}}{4 \pi s}\right)^{d / 2} e^{-\frac{1}{2}\left(|x|^{2}+|y|^{2}\right)}\right) f(y) d y \\
& \times\left(\log \frac{1+s}{1-s}\right)^{-1 / 2} \frac{d s}{1-s^{2}}
\end{aligned}
$$

where $K_{S}$ is the kernel defined in (9). Analogous considerations can be made with $e^{-t(\mathcal{L}-d)}=e^{t d} e^{-t \mathcal{L}}$ and we can write

$$
\begin{aligned}
((\mathcal{L} & \left.-d)^{-1 / 2}-\mathcal{L}^{-1 / 2}\right) f(x) \\
= & \sqrt{\frac{2}{\pi}} \int_{0}^{1}\left\{\left(\frac{1+s}{1-s}\right)^{d / 2}-1\right\} \\
& \times \int_{\mathbb{R}^{n}}\left(K_{s}(x, y)-\chi_{(1 / 2,1)}(s)\left(\frac{1-s^{2}}{4 \pi s}\right)^{d / 2} e^{-\frac{1}{2}\left(|x|^{2}+|y|^{2}\right)}\right) f(y) d y \\
& \times\left(\log \frac{1+s}{1-s}\right)^{-1 / 2} \frac{d s}{1-s^{2}}:=N(x, y) .
\end{aligned}
$$

We shall see that the function $N(x, y)$ just defined satisfies the theorem. Write

$$
N(x, y)=\int_{0}^{1 / 2}+\int_{1 / 2}^{1}=I_{0}+I_{1}
$$


Observe that for $s \in(0,1 / 2)$ we have $\left(\frac{1+s}{1-s}\right)^{d / 2}-1 \sim s$ and $\log \frac{1+s}{1-s} \sim s$ therefore

$$
I_{0} \leq C \int_{0}^{1 / 2} s^{1 / 2} \frac{1}{s^{d / 2}} e^{-\frac{c}{s}|x-y|^{2}} d s \leq \frac{C}{|x-y|^{d-3}} \int_{c_{0}|x-y|^{2}}^{\infty} u^{\frac{d-3}{2}} e^{-u} \frac{d u}{u} .
$$

In the last inequality we performed the change of variables $u=c \frac{|x-y|^{2}}{s}$. If $c_{0}|x-y|>1$, by using the inequality $z^{n} e^{-z} \leq C e^{-z / 2}$, we get

$$
I_{0} \leq \frac{C}{|x-y|^{d-3}} e^{-\frac{|x-y|^{2}}{c}} \int_{c}^{\infty} u^{\frac{d-3}{2}} e^{-u / 2} \frac{d u}{u} \leq C e^{-\frac{|x-y|^{2}}{c}} .
$$

On the other hand, if $c_{0}|x-y|<1$ and $d \geq 4$ we have

$$
I_{0} \leq \frac{C}{|x-y|^{d-3}}\left(\int_{c_{0}|x-y|^{2}}^{1}+\int_{1}^{\infty}\right) u^{\frac{d-3}{2}} e^{-u} \frac{d u}{u} \leq \frac{C}{|x-y|^{d-3}} .
$$

If $c_{0}|x-y|<1$ and $d<4$ we have

$$
\begin{aligned}
I_{0} & \leq \frac{C}{|x-y|^{d-3}}\left(\int_{c_{0}|x-y|^{2}}^{1}+\int_{1}^{\infty}\right) u^{\frac{d-3}{2}} e^{-u} \frac{d u}{u} \leq \frac{C}{|x-y|^{d-3}}\left(\int_{c_{0}|x-y|^{2}}^{1} u^{\frac{d-3}{2}} e^{-u} \frac{d u}{u}+C\right) \\
& \leq C\left(\int_{c_{0}|x-y|^{2}}^{1} e^{-u} \frac{d u}{u}+C\right) \leq C(\log |x-y|+1) .
\end{aligned}
$$

Where we have used $\left(\frac{u}{|x-y|^{2}}\right)^{d-3} \leq C$, valid for $d \leq 3$.

On the other hand we write $I_{1}=I_{11}+I_{12}$ where

$$
\begin{aligned}
I_{12}= & \sqrt{\frac{2}{\pi}} \int_{1 / 2}^{1}\left[\left(\frac{1+s}{1-s}\right)^{d / 2} K_{s}(x, y)-\left(\frac{1+s}{4 \pi s}\right)^{d / 2} \exp \left(-\frac{|x|^{2}+|y|^{2}}{2}\right)\right] \\
& \times\left(\log \frac{1+s}{1-s}\right)^{-1 / 2} \frac{d s}{1-s^{2}} .
\end{aligned}
$$

Consider the function $\beta(\theta)=\exp \left(-\frac{1}{4}\left(\theta|x+y|^{2}+\frac{1}{\theta}|x-y|^{2}\right)\right.$. Since $\frac{1}{2}<s<1$, applying the mean value theorem we have

$$
\mid \exp \left(-\frac{1}{4}\left(s|x+y|^{2}+\frac{1}{s}|x-y|^{2}\right)-\exp \left(-\frac{|x|^{2}+|y|^{2}}{2}\right) \mid \leq C e^{-\frac{|x-y|^{2}}{c}}(1-s) .\right.
$$

Hence

$$
I_{12} \leq C e^{-\frac{|x-y|^{2}}{c}} \int_{1 / 2}^{1}(1-s)\left(\log \frac{1+s}{1-s}\right)^{-1 / 2} \frac{d s}{1-s} \leq C e^{-\frac{|x-y|^{2}}{c}} .
$$

The case $I_{11}$ is similar. In order to prove part (ii) of the Theorem we consider the kernel

$$
A_{i} N(x, y)=\left(\frac{\partial}{\partial x_{i}}-x_{i}\right) N(x, y)=\frac{\partial}{\partial x_{i}} N(x, y)-x_{i} N(x, y)=N_{1}(x, y)-N_{2}(x, y) .
$$

In order to handle these kernels we follow the procedure we did for $N$, that is, consider separately the cases $0 \leq s \leq 1 / 2$ and $1 / 2 \leq s \leq 1$. We shall estimate first the kernel $N_{2}$. 
We call again $I_{0}$ the integral in the range $0 \leq s \leq 1 / 2$. If $x \cdot y \leq 0$ then $|x| \leq|x-y|$, therefore by using $z^{n} e^{-z} \leq C e^{-z / 2}$ we have

$$
\begin{aligned}
I_{0} & \leq C \int_{0}^{1 / 2}|x-y| s^{1 / 2} \frac{1}{s^{d / 2}} e^{-\frac{|x-y|^{2}}{c s}} d s \leq C \int_{0}^{1 / 2} s \frac{1}{s^{d / 2}} e^{-\frac{|x-y|^{2}}{c s}} d s \\
& \leq \frac{C}{|x-y|^{d-4}} \int_{c|x-y|^{2}}^{\infty} u^{\frac{d-4}{2}} e^{-u} \frac{d u}{u} .
\end{aligned}
$$

In the last inequality we performed the change of variables $u=c \frac{|x-y|^{2}}{s}$. If $c|x-y|>1$, by using the inequality $z^{n} e^{-z} \leq C e^{-z / 2}$, we get

$$
I_{0} \leq \frac{C}{|x-y|^{d-4}} e^{-\frac{|x-y|^{2}}{c}} \int_{c}^{\infty} u^{\frac{d-4}{2}} e^{-u / 2} \frac{d u}{u} \leq C e^{-\frac{|x-y|^{2}}{c}} .
$$

On the other hand, if $c|x-y|<1$ and $d \geq 5$ we have

$$
I_{0} \leq \frac{C}{|x-y|^{d-4}}\left(\int_{c_{0}|x-y|^{2}}^{1}+\int_{1}^{\infty}\right) u^{\frac{d-4}{2}} e^{-u} \frac{d u}{u} \leq \frac{C}{|x-y|^{d-4}} .
$$

If $c_{0}|x-y|<1$ and $d<5$ we have

$$
\begin{aligned}
I_{0} & \leq \frac{C}{|x-y|^{d-4}}\left(\int_{c_{0}|x-y|^{2}}^{1}+\int_{1}^{\infty}\right) u^{\frac{d-4}{2}} e^{-u} \frac{d u}{u} \leq \frac{C}{|x-y|^{d-4}}\left(\int_{c_{0}|x-y|^{2}}^{1} u^{\frac{d-4}{2}} e^{-u} \frac{d u}{u}+C\right) \\
& \leq C\left(\int_{c_{0}|x-y|^{2}}^{1} e^{-u} \frac{d u}{u}+C\right) \leq C(\log |x-y|+1) .
\end{aligned}
$$

where we have used that since $d \leq 4$ then $\left(\frac{u}{|x-y|^{2}}\right)^{d-4} \leq C$. If $x \cdot y \geq 0$ then $|x| \leq|x+y|$, therefore we have (we use the term $e^{s|x+y|^{2}}$ in $K_{s}$ and the fact $s^{1 / 2}|x+y| e^{-s|x+y|^{2}} \leq C$ )

$$
I_{0} \leq C \int_{0}^{1 / 2} \frac{1}{s^{d / 2}} e^{-\frac{|x-y|^{2}}{c s}} d s \leq \frac{C}{|x-y|^{d-2}} \int_{c|x-y|^{2}}^{\infty} u^{\frac{d-2}{2}} e^{-u} \frac{d u}{u} .
$$

In the last inequality we performed the change of variables $u=c \frac{|x-y|^{2}}{s}$. We proceed analogously to the case $x \cdot y \leq 0$. Pasting up the above arguments with the arguments we gave above for the integral $I_{1}$ (in the range $1 / 2 \leq s \leq 1$ ) for $N$ we get in this case $I_{1} \leq C e^{-\frac{|x-y|^{2}}{c}}$. This completes the proof for the kernel $N_{2}$.

Now we shall analyze the kernel $N_{1}=\frac{\partial}{\partial x_{i}} N(x, y)$. Observe that

$$
\begin{aligned}
\frac{\partial}{\partial x_{i}} N(x, y)= & \sqrt{\frac{2}{\pi}} \int_{0}^{1}\left(\log \frac{1+s}{1-s}\right)^{-1 / 2}\left\{\left(\frac{1+s}{1-s}\right)^{d / 2}-1\right\} \\
& \times\left[\left(-\frac{1}{2}\left(s\left(x_{i}+y_{i}\right)+\frac{x_{i}-y_{i}}{s}\right)\right) K_{s}(x, y)+\chi_{[1 / 2,1]}(s)\left(\frac{1-s^{2}}{4 \pi s}\right)^{d / 2}\right. \\
& \left.\times x_{i} \exp \left(-\frac{1}{2}\left(|x|^{2}+|y|^{2}\right)\right)\right] \frac{d s}{1-s^{2}} \\
= & \sqrt{\frac{2}{\pi}}\left(\int_{0}^{1 / 2}+\int_{0}^{1 / 2} \ldots d s\right)=I_{1}+I_{2} .
\end{aligned}
$$


Observe that $\left|\left(-\frac{1}{2}\left(s\left(x_{i}+y_{i}\right)+\frac{x_{i}-y_{i}}{s}\right)\right)\right| \leq\left(\frac{1}{2}\left(s|x+y|+\frac{|x-y|}{s}\right)\right)$. We consider again separately the case $0 \leq s \leq 1 / 2$ and denoting $I_{0}$ the corresponding integral, we have

$$
I_{0} \leq C \int_{0}^{1 / 2}\left(s|x+y|+\frac{1}{s}|x-y|\right) s^{1 / 2} \frac{1}{s^{d / 2}} e^{-\frac{1}{4}\left(s|x+y|^{2}+\frac{1}{s}|x-y|^{2}\right)} d s .
$$

The same arguments used for $N_{2}$ can be repeated to get the required bound for $N_{1}$ in this case.

As for $I_{1}$ we can proceed analogously by considering the function

$$
\beta(\theta)=\left(-\frac{1}{2}\left(\theta\left(x_{i}+y_{i}\right)+\frac{1}{\theta}\left(x_{i}-y_{i}\right)\right)\right) \exp \left(-\frac{1}{4}\left(\theta|x+y|^{2}+\frac{1}{\theta}|x-y|^{2}\right) .\right.
$$

Since $\frac{1}{2}<s<1$, applying the mean value theorem we have

$$
\begin{gathered}
\mid\left(-\frac{1}{2}\left(s\left(x_{i}+y_{i}\right)+\frac{1}{s}\left(x_{i}-y_{i}\right)\right)\right) \exp \left(-\frac{1}{4}\left(s|x+y|^{2}+\frac{1}{s}|x-y|^{2}\right)\right) \\
+x_{i} \exp \left(-\frac{|x|^{2}+|y|^{2}}{2}\right)|=| \beta(s)-\beta(1) \mid \leq C e^{-\frac{|x-y|^{2}}{c}}(1-s) .
\end{gathered}
$$

Again the arguments given for $N_{1}$ and $N$ are valid in this case.

4. Proofs of the main results. We begin this section by presenting the proof of Theorem 2.1. This theorem will be obtained by using the following theorem, that can be found in [6].

THEOREM 4.1. Let $v$ be a positive measurable function. The following conditions are equivalent:

(i) There exists a positive measurable function $u$ and a constant $C$ such that for every $f \in L^{2}(v(x) d x)$ we have $\sup _{t} \int_{\mathbb{R}^{d}}\left|e^{-t \mathbf{L}} f(x)\right|^{2} u(x) d \gamma(x) \leq C \int_{\mathbb{R}^{d}}|f(x)|^{2} v(x) d \gamma(x)$.

(ii) There exists a positive measurable function $u$ and a constant $C$ such that for every $f \in L^{2}(v(x) d \gamma(x))$ we have

$\int_{\mathbb{R}^{d}} \sup _{t}\left|e^{-t \mathbf{L}} f(x)\right|^{2} u(x) d x \leq C \int_{\mathbb{R}^{d}}|f(x)|^{2} v(x) d \gamma(x)$.

(iii) The function $v$ satisfies $\int_{\mathbb{R}^{d}} v^{-1}(x) d \gamma(x)<\infty$.

Observe that by using Proposition 3.3 (iii) and Lemma 3.1 we have

$$
\begin{aligned}
\left\|e^{-t(\mathcal{L}-d)} f\right\|_{L^{2}(u(x) d x)} & =\left\|U^{-1} e^{-t(\mathcal{L}-d)} f\right\|_{L^{2}(u(x) d \gamma(x))}=\left\|e^{-t(\mathbf{L})} U^{-1} f\right\|_{L^{2}(u(x) d \gamma(x))} \\
& \leq C\left\|U^{-1} f\right\|_{L^{2}(v(x) d \gamma(x))}=\|f\|_{L^{2}(v(x) d x)}
\end{aligned}
$$

were in the penultimate inequality we have used Theorem 4.1. In order to finish the proof of Theorem 2.1 observe that for each $t$ and each $x$ we have $e^{-t(\mathcal{L})} f(x) \leq$ $e^{-t(\mathcal{L}-d)} f(x)$.

We continue by presenting the proof of Theorem 2.2. If $f$ is a linear combination of Hermite functions with $\int_{\mathbb{R}^{n}} f(y) e^{-\frac{|y|^{2}}{2}} d y=0$, then $f=U g$ and $g(y)=U^{-1} f(y)=$ $f(y) e^{\frac{|y|^{2}}{2}} \pi^{-d / 4}$ (where $U$ is the isometry in Lemma 3.1 and $g$ is a scalar valued polynomial function with $\left.\int g(y) d \gamma(y)=0\right)$. Then as we mention in the introduction, the Riesz 
transforms associated to the Ornstein-Uhlenbeck differential operator are principal value operators, therefore by using Proposition 3.3(v), we have

$$
\begin{aligned}
A_{j}(\mathcal{L}-d)^{-1 / 2} f(x) & =U \delta_{j}(\mathbf{L})^{-1 / 2} g(x)=U \mathbf{R}_{j} g(x) \\
& =e^{-\frac{|x|^{2}}{2}} \pi^{d / 4} \lim _{\varepsilon \rightarrow 0} \int_{|x-y|>\varepsilon} \mathbf{R}_{j}(x, y) f(y) e^{\frac{|y|^{2}}{2}} \pi^{-d / 4} d \gamma(y) \\
& =\pi^{-d / 2} \lim _{\varepsilon \rightarrow 0} \int_{|x-y|>\varepsilon} \mathbf{R}_{j}(x, y) f(y) e^{-\frac{|x|^{2}}{2}} e^{\frac{-|y|^{2}}{2}} d y .
\end{aligned}
$$

If $h$ is a linear combination of Hermite functions and $\int_{\mathbb{R}^{n}} h(y) e^{-|y|^{2} / 2} d y=0$, then the conclusion of the theorem for the operator $\mathcal{R}_{i}^{+}$, follows from Theorem 3.5(ii). For a general $f$ linear combination of Hermite functions we have $f(x)=$ $h(x)+\pi^{-d / 2} e^{-|x|^{2} / 2}\left(\int_{\mathbb{R}^{n}} f(y) e^{-|y|^{2} / 2} d y\right)=h(x)+c h_{0}(x)$ with $\int_{\mathbb{R}^{n}} h(y) e^{-|y|^{2} / 2}=0$ and $h_{0}$ is the first Hermite function. Therefore, as $A_{i}\left(h_{0}\right)=0$, we have $\mathcal{R}_{i}^{+} f=A_{i}(\mathcal{L})^{-1 / 2} f=$ $A_{i}(\mathcal{L})^{-1 / 2} h+c A_{i}(\mathcal{L})^{-1 / 2}\left(h_{0}\right)=\mathcal{R}_{i}^{+} h$. Then the theorem follows for $\mathcal{R}_{i}^{+}$. Observe that since $\mathcal{L}^{-1 / 2}$ is given by an integrable kernel, see Proposition 3.4 , the operator $x_{i} \mathcal{L}^{-1 / 2}$ is a principal value operator. Therefore as $\mathcal{R}_{i}^{-}=-\mathcal{R}_{i}^{+}+2 x_{i} \mathcal{L}^{-1 / 2}$ we get the desired result for $\mathcal{R}_{i}^{-}$. Once we get the conclusion for $\mathcal{R}_{i}^{-}$we use again Theorem 3.5 and Proposition 3.3 and we obtain the conclusion for $\delta_{i}^{*} \mathbf{L}^{-1 / 2} \Pi_{0}$.

Proof of Theorem 2.5. Observe that given a function $f \in L^{2}\left(\mathbb{R}^{n}, d x\right)$ then

$$
f(x)=g(x)+\pi^{-1 / 2} e^{-|x|^{2} / 2}\left(\int_{\mathbb{R}^{n}} f(y) e^{-|y|^{2} / 2} d y\right)=g(x)+P_{0}(f)(x),
$$

and $\int_{\mathbb{R}^{d}} g(x) e^{-|x|^{2} / 2} d x=0$. Clearly $\mathcal{R}_{j}^{+} f=\mathcal{R}_{j}^{+} g$ and

$$
\mathcal{R}_{j}^{-} f=\mathcal{R}_{j}^{-} g+c p(x) e^{-|x|^{2} / 2}\left(\int_{\mathbb{R}^{n}} f(y) e^{-|y|^{2} / 2} d y\right),
$$

where $p(x)$ is a polynomial de degree one in $x$. As we said in the introduction, see [15], the operators $\mathcal{R}_{i}^{ \pm}, i=1, \ldots, n$ are bounded in $L^{2}\left(\mathbb{R}^{n}, \omega(x) d x\right)$ for any weight $\omega$ which belongs to the $A_{2}$ Muckenhoupt class. In particular

$$
\left\|\mathcal{R}_{j}^{+} g\right\|_{L^{2}\left(\mathbb{R}^{d}, \omega(x) d x\right)}^{2} \leq\|g\|_{L^{2}\left(\mathbb{R}^{d}, \omega(x) d x\right)}^{2}
$$

It is well known that the Hardy-Littlewood maximal operator $M$ maps $L^{2}\left(\mathbb{R}^{n}, \omega(x) d x\right)$ into itself, again for $\omega \in A_{2}$. Therefore Theorem 3.5 says that the difference $A_{i}(\mathcal{L}-$ $d)^{-1 / 2}-\mathcal{R}_{i}^{+}$maps $L^{2}\left(\mathbb{R}^{n}, \omega(x) d x\right)$ into itself. Then $A_{i}(\mathcal{L}-d)^{-1 / 2}$ maps $L^{2}\left(\mathbb{R}^{n}, \omega(x) d x\right)$, we get the result for $\mathbf{R}_{i}$ by using Proposition 3.3 and Lemma 3.1.

On the other hand it is well known that if $v$ is a weight which belongs to the $A_{2}$ Muckenhoupt class then the measure $v(x) d x$ is doubling, that is there exists a constant such that $\int_{\{|x|<2 r\}} v(x) d x \leq A \int_{\{|x|<r\}} v(x) d x$ therefore, for any $\varepsilon>0$, we have

$$
\begin{aligned}
\int_{\mathbb{R}^{n}} v(y) e^{-\varepsilon|y|^{2}} d y & \leq \sum_{j=0}\left(\int_{2^{j}<|y|<2^{j+1}} v(y) e^{-\varepsilon|y|^{2}} d y\right)+\int_{|y|<1} v(y) e^{-\varepsilon|y|^{2}} d y \\
& \leq \sum_{j=0} e^{-\varepsilon 2^{2 j}}\left(\int_{2^{j}<|y|<2^{j+1}} v(y) d y\right)+\int_{|y|<1} v(y) d y \\
& \leq \sum_{j=0} e^{-\varepsilon 2^{2 j}} A^{j} \int_{|y|<1} v(y) d y \leq C_{\varepsilon}(\nu) .
\end{aligned}
$$


By using $|p(x)| e^{-|x|^{2}} \leq C e^{-|x|^{2} / 2}$ and the fact that if $\omega \in A_{2}$ then $\omega^{-1} \in A_{2}$ we have

$$
\begin{aligned}
& \int_{\mathbb{R}^{d}} p(x)^{2}\left(\int_{\mathbb{R}^{d}} f(y) e^{-|y|^{2} / 2} d y\right)^{2} e^{-|x|^{2}} \omega(x) d x \\
& \quad \leq C \int_{\mathbb{R}^{d}}\left(\int_{\mathbb{R}^{d}}|f(y)|^{2} \omega(y) d y\right)\left(\int_{\mathbb{R}^{d}} \omega(y)^{-1} e^{-|y|^{2}} d y\right) e^{-|x|^{2} / 2} \omega(x) d x \\
& \quad \leq C\left(\int_{\mathbb{R}^{d}}|f(y)|^{2} \omega(y) d y\right)\left(\int_{\mathbb{R}^{d}} \omega(y)^{-1} e^{-|y|^{2}} d y\right)\left(\int_{\mathbb{R}^{d}} \omega(x) e^{-|x|^{2} / 2} d x\right) \\
& \leq C\left(\int_{\mathbb{R}^{d}}|f(y)|^{2} \omega(y) d y\right) .
\end{aligned}
$$

Therefore, by using (13) and the fact that $\mathcal{R}_{i}^{-}, i=1, \ldots, n$ are bounded in $L^{2}\left(\mathbb{R}^{n}, \omega(x) d x\right)$, we have that

$$
\left\|\mathcal{R}_{j}^{-} g\right\|_{L^{2}\left(\mathbb{R}^{d}, \omega(x) d x\right)}^{2} \leq\|g\|_{L^{2}\left(\mathbb{R}^{d}, \omega(x) d x\right)}^{2} .
$$

In order to get the result for $\delta^{*}(\mathbf{L})^{-1 / 2} \Pi_{0}$ we can now proceed as with $\mathbf{R}_{i}$.

Proof of Theorem 2.3. We call $\mathbf{R}_{i}$ the linear extension of these operators, in the sense described in (4), to functions taking values in a Banach space $B$. The following theorem was proved in [7].

THEOREM 4.2. The following statements are equivalent: $(i) \mathbf{R}_{i}, i=1, \ldots, n$ are $L_{B}^{p}\left(\mathbb{R}^{n}, d \gamma\right)$ bounded for every $p, 1<p<\infty$. (ii) $\mathbf{R}_{i}, i=1, \ldots, n$ are $L_{B}^{p}\left(\mathbb{R}^{n}, d \gamma\right)$ bounded for a particular $p, 1<p<\infty$. (iii) $\mathbf{R}_{i}, i=1, \ldots, n$ are bounded from $L_{B}^{1}\left(\mathbb{R}^{n}, d \gamma\right)$ into $L_{B}^{1, \infty}\left(\mathbb{R}^{n}, d \gamma\right)$. (iv) B has the UMD property.

It was proved in [15] that the operators $\mathcal{R}_{j}^{ \pm}$are Calderón-Zygmund operators with associated kernels $\mathcal{R}_{j}^{ \pm}(x, y)$, in these circumstances it is known that (ii), (iii), (iv), (v) are equivalent, where in (iv) $L^{\infty}$ has to be substituted by $L_{c}^{\infty}$. The proof of this fact consists in adapting the scalar case to this vector valued case. For the scalar case see [8]. These equivalences have as a consequence that any of them is equivalent to the following statement:

(iii) $^{\prime}$ There exists a constant $C_{2}$ such that $\left\|\mathcal{R}_{j}^{+} f\right\|_{L_{B}^{2}(d x)} \leq C_{2}\|f\|_{L_{B}^{2}(d x)}, \quad 1 \leq j \leq n$.

Since by Theorem 3.5, the difference between $\mathcal{R}_{j}^{+}$and $A_{j}(\mathcal{L}-d)^{-1 / 2}$ is controlled by a positive operator bounded in $L^{p}, 1 \leq p \leq \infty$, we have that in (iii)' we can replace $\mathcal{R}_{j}^{+}$by $A_{j}(\mathcal{L}-d)^{-1 / 2}$. Now by using Lemma 3.1 we see that (iii)' is equivalent to (iii)" There exists a constant $C_{2}$ such that $\left\|\mathbf{R}_{j} f\right\|_{L_{B}^{2}(d \gamma)} \leq C_{2}\|f\|_{L_{B}^{2}(d \gamma)}, \quad 1 \leq j \leq n$.

But statement (iii)" is equivalent to say that the Banach space $B$ is $U M D$, see Theorem 4.2.

By using Theorem 2.2 and the vector valued version of the general theory of Calderón-Zygmund operators, [8], [11], we get

$$
\mathcal{R}_{j}^{+} f(x)=\lim _{\varepsilon \rightarrow 0} \mathcal{R}_{j, \varepsilon}^{+} f(x)=\lim _{\varepsilon \rightarrow 0} \int_{|x-y|>\varepsilon} \mathcal{R}_{j}^{+}(x, y) f(y) d y, \quad \text { a.e. } x, \quad f \in \cup_{1 \leq p<\infty} L_{B}^{p} .
$$

Now we can use Remark 2.4 and we get the result for $L^{\infty}$. The theorem for $\mathcal{R}_{j}^{-}$follows by observing that $\mathcal{R}_{j}^{-}=-\mathcal{R}_{j}^{+}+2 x_{i} \mathcal{L}^{-1 / 2}$. 


\section{REFERENCES}

1. J. Bourgain, Some remarks on Banach spaces in which martingale differences are unconditional, Arkiv. Mat. 21 (1983), 163-168.

2. D. Burkholder, A geometric condition that implies the existence of certain singular integrals of Banach-space-valued functions, Conference Harmonic Analysis in Honor of A. Zygmund (W. Beckner, A.P. Calderón, R. Fefferman and P. W. Jones, eds) (Wadswoth Inc., Belmont,CA, 1981), 270-286. 2001).

3. J. Duoandikoetxea, Fourier analysis (American Mathematical Society, Providence,

4. E. B. Fabes, C. E. Gutiérrez and R. Scotto, Weak-type estimates for the Riesz transforms associated with the Gaussian measure, Revista Matemática Iberoamericana 10 (1994) No. 2, $229-281$. $107-134$.

5. C. Gutiérrez, On the Riesz transforms for Gaussian measures, J. Funct. Anal. 120 (1994),

6. E. Harboure, J. L. Torrea and B. Viviani, On the search for weighted inequalities for operators realted to the Ornstein-Uhlenbeck semigroup, Math. Ann. 318 (2000), 341-353.

7. E. Harboure, J. L. Torrea and B. Viviani, Vector-valued extensions of operators related to the Ornstein-Uhlenbeck semigroup, J. d'Analyse Math. 91 (2003), 1-29.

8. J. L. Journé, Calderón-Zygmund operators, pseudo-differential operators, and the Cauchy integral of Calderón, Lecture Notes in Mathematics, No. 994 (Springer-Verlag, 1983).

9. B. Muckenhoupt, Poisson integrals for Hermite and Laguerre expansions, Trans. Amer. Math. Soc. 139 (1969), 231-242.

10. B. Muckenhoupt, Hermite conjugate expansions, Trans. Amer. Math. Soc. 139 (1969), $243-260$.

11. J. L. Rubio de Francia, F. J. Ruiz and J. L. Torrea, Calderón Zygmund theory for operator-valued kernels, Adv. in Math. 62 (1986), 7-48.

12. P. Sjögren, On the maximal function for the Mehler kernel, in Harmonic Analysis, Cortona 1982, Lecture Notes in Mathematics No. 992 (Springer-Verlag, 1983), 73-82.

13. E. M. Stein, Topics in Harmonic Analysis Related to the Littlewood-Paley Theory (Princeton University Press, 1970).

14. E. M. Stein, Harmonic analysis real-variable methods, orthogonality, and oscillatory integrals, Princeton Mathematical Series 43, Princeton, New Jersey, 1993.

15. K. Stempak and J. L. Torrea, Poisson integrals and Riesz transforms for Hermite function expansions with weights, J. Funct. Anal. 202 (2003), 443-472.

16. K. Stempak and J. L. Torrea, BMO results for operators associated to the Hermite function expansions. Preprint 2005.

17. P. Sjögren, Operators associated with the Hermite semigroup-a survey.

18. G. Szegö, Orthogonal polynomials, American Mathematical Society Colloquium Publications, Vol. 23 (American Mathematical Society, 1939).

19. S. Thangavelu, Lectures on Hermite and Laguerre expansions, Math. Notes 42 (Princeton University Press, 1993). 